\title{
Electrophysiological study of accessory deep peroneal nerve in a sample of Egyptian subjects Emmanuel K.A. Saba
}

Department of Physical Medicine, Rheumatology and Rehabilitation, Faculty of Medicine, Alexandria University, Alexandria, Egypt

Correspondence to Emmanuel K.A. Saba, MD Department of Physical Medicine,

Rheumatology and Rehabilitation, Faculty of Medicine, Alexandria University, Alexandria, 21131, Egypt. Tel: 0201224718376;

e-mail: emadaziz55@yahoo.com

Received 21 February 2019

Accepted 9 April 2019

Egyptian Rheumatology \& Rehabilitation 2019, 46:251-256

\begin{abstract}
Introduction
Accessory deep peroneal nerve (ADPN) is the most common anomalous innervation present in the lower limb.

Aim

The aim of this study was to determine the prevalence of ADPN electrophysiologically in a sample of healthy Egyptian individuals.

Subjects and methods

This cross-sectional study included 200 lower limbs from 100 [56 (56\%) women and $44(44 \%)$ men] Egyptian apparently healthy volunteers. Motor nerve conduction studies for the peroneal nerve and ADPN were done.

Results

ADPN was found in $20(10 \%)$ lower limbs of $17(17 \%)$ subjects. There was no statistically significant difference between the occurrence of ADPN in women versus men $(P=0.797)$. ADPN was found in $11(55 \%)$ right lower limbs and in nine (45\%) left lower limbs. There was no statistically significant difference between the occurrence of ADPN in right lower limbs versus left lower limbs among subjects with ADPN $(P=0.637)$. It was present bilaterally in three $(17.65 \%)$ subjects. There was no statistically significant difference between the occurrence of bilateral ADPN in women versus men $(P=0.761)$. Among the $14(82.35 \%)$ subjects with unilateral ADPN, it was present in the right side in eight $(57.14 \%)$ subjects. There was no statistically significant difference between the occurrence of unilateral ADPN in the right side versus left side $(P=0.579)$.

Conclusion

This study demonstrated that ADPN prevalence in the referred Egyptian sample through electrodiagnostic studies of lower limbs was $17 \%$, with no sex nor side difference. Recognition of ADPN is essential for proper interpretation of lower limbs electrophysiological data.
\end{abstract}

\section{Keywords: \\ Egypt Rheumatol Rehabil 46:251-256 \\ (1) 2019 Egyptian Society for Rheumatology and Rehabilitation \\ 1110-161X}

accessory deep peroneal nerve, anomalous innervations, Egyptian population

\section{Introduction}

Peripheral nerves anomalous innervations are essential aspects in routine electrophysiological assessment [1]. Accessory deep peroneal nerve (ADPN) is the most common anomalous innervation present in the lower limbs [1-4]. The ADPN should be taken into consideration during interpretation of patients with suspected common peroneal, deep peroneal, and superficial peroneal nerve lesions clinically and electrophysiologically $[1,3,5-8]$. It is a branch of the superficial peroneal nerve, the continuation of the muscular branch that supplies the peroneus longus and peroneus brevis muscles [2]. The ADPN gives motor supply to the lateral aspect of the extensor digitorum brevis (EDB) muscle while the deep peroneal nerve supplies the medial aspect of it (Fig. 1) $[8,9]$. It was reported that ADPN was present in $12-35 \%$ of the population [10].

Up to our knowledge, no study about the prevalence of ADPN among Egyptian subjects have been published till now. As it is essential and critical, the aim of this study was to determine the prevalence of ADPN electrophysiologically in a sample of healthy Egyptian individuals.

\section{Subjects and methods}

This cross-sectional study included 200 lower limbs of 100 apparently healthy Egyptian volunteers. Lack of neurological symptoms and normal neurological examination of both lower limbs were the inclusion criteria for the subjects. The volunteers included medical staff, their relatives, and relatives of patients attending the outpatient clinic of Physical Medicine, Rheumatology and Rehabilitation Department, Main University Hospital, Alexandria Faculty of Medicine.

This is an open access journal, and articles are distributed under the terms of the Creative Commons Attribution-NonCommercial-ShareAlike 4.0 License, which allows others to remix, tweak, and build upon the work non-commercially, as long as appropriate credit is given and the new creations are licensed under the identical terms. 
The research was explained thoroughly, then an informed consent was given by each participant. The Ethics Committee of the Faculty of Medicine, Alexandria University, Egypt had approved the study.

Demographic data were collected from all studied subjects. Neurological examination was done for all of them.

Electrophysiological studies were conducted on a Nihon Kohden Neuropack S1 MEB-9400 unit with a two-channel evoked potential/EMG measuring system (Nihon Kohden Corporation, Tokyo, Japan). Temperature of the skin (at the site of the recording electrodes) was maintained at around $32-34^{\circ} \mathrm{C}$ by means of an infrared lamp. The ground electrode was placed between the stimulation site proximally and the recording electrodes distally [1]. A measurement tape was used for measuring the conduction distances with a precision of $1 \mathrm{~mm}$.

The following parameters were applied for the motor nerve conduction studies: The filter bandwidth was 10 $\mathrm{Hz}-10 \mathrm{kHz}$. The pulse duration was $0.2 \mathrm{~ms}$. The current production ability of the bipolar stimulator was $50 \mathrm{~mA}$. The compound muscle action potentials (CMAP) amplitude was measured from the baseline to the negative peak expressed in millivolts. Supramaximal stimulation was ensured. The electrophysiological studies were done by caution. It was essential to make sure that the change in the CMAP amplitude obtained between distal and proximal sites of stimulation was not due to technical factors such as submaximal stimulation and co-stimulation of the nearby nerves [1].

The peroneal motor nerve conduction study and ADPN motor nerve conduction study recording the EDB muscle were done as the following: the active recording surface disc electrode was placed over the EDB muscle belly on the dorsolateral aspect of the foot and the reference surface disc electrode was placed over the dorsal aspect of the metatarsophalangeal joint of the fifth toe (Figs 2 and 3) [1].

Electrical stimulation of the peroneal nerve was done at the following sites: (i) distal stimulation: at $9 \mathrm{~cm}$ proximal to the active recording electrode at the ankle slightly lateral to the tibialis anterior tendon; (ii) proximal stimulation at two sites: the first site is below fibular head on the lateral calf at about twofinger breadths inferior to the fibular head, and the second site is at the lateral popliteal fossa adjacent to the external hamstring tendons at a distance of $10 \mathrm{~cm}$ proximal to the below fibular head site of stimulation (Fig. 2). The sweep speed was $5 \mathrm{~ms} /$ division and the sensitivity was $5 \mathrm{mV} /$ division. The CMAP amplitude was taken for analysis [1].

Criteria for suspecting the presence of ADPN electrophysiologically included the following [1,11]:

(1) The peroneal CMAP amplitude recorded from the EDB muscle was higher at the below fibular head and lateral popliteal fossa sites of stimulation than that obtained at the ankle site of stimulation.

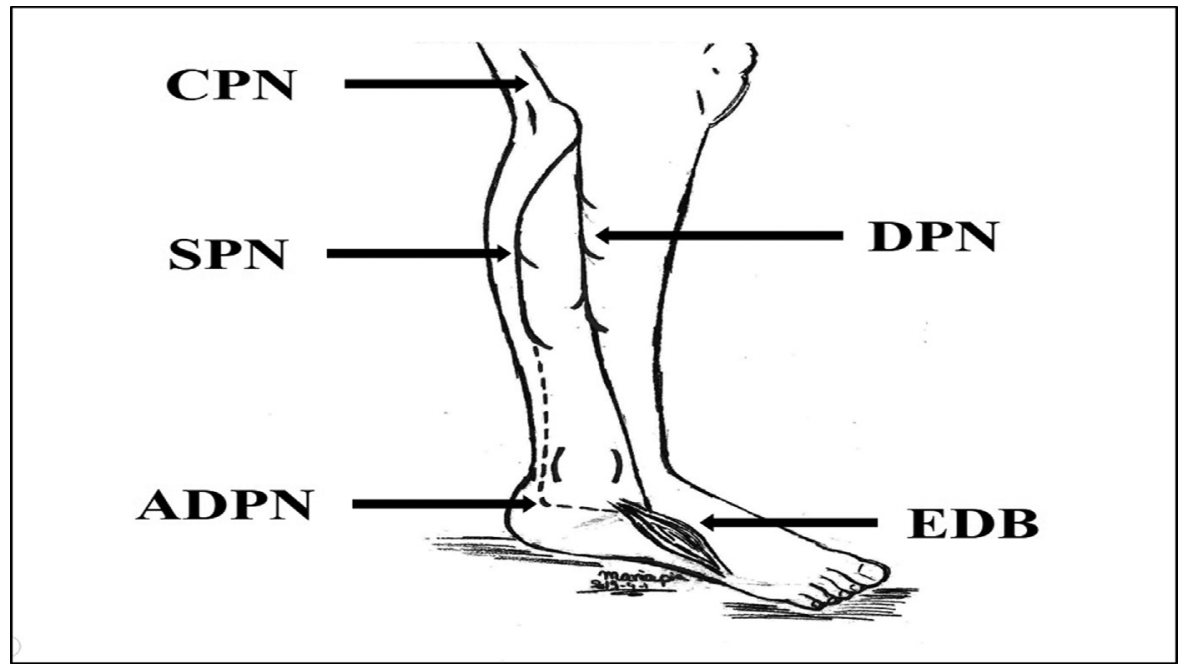

Illustration representing the common peroneal nerve (CPN), deep peroneal nerve (DPN), superficial peroneal nerve (SPN), accessory deep peroneal nerve (ADPN), and extensor digitorum brevis (EDB) muscle. 
Figure 2

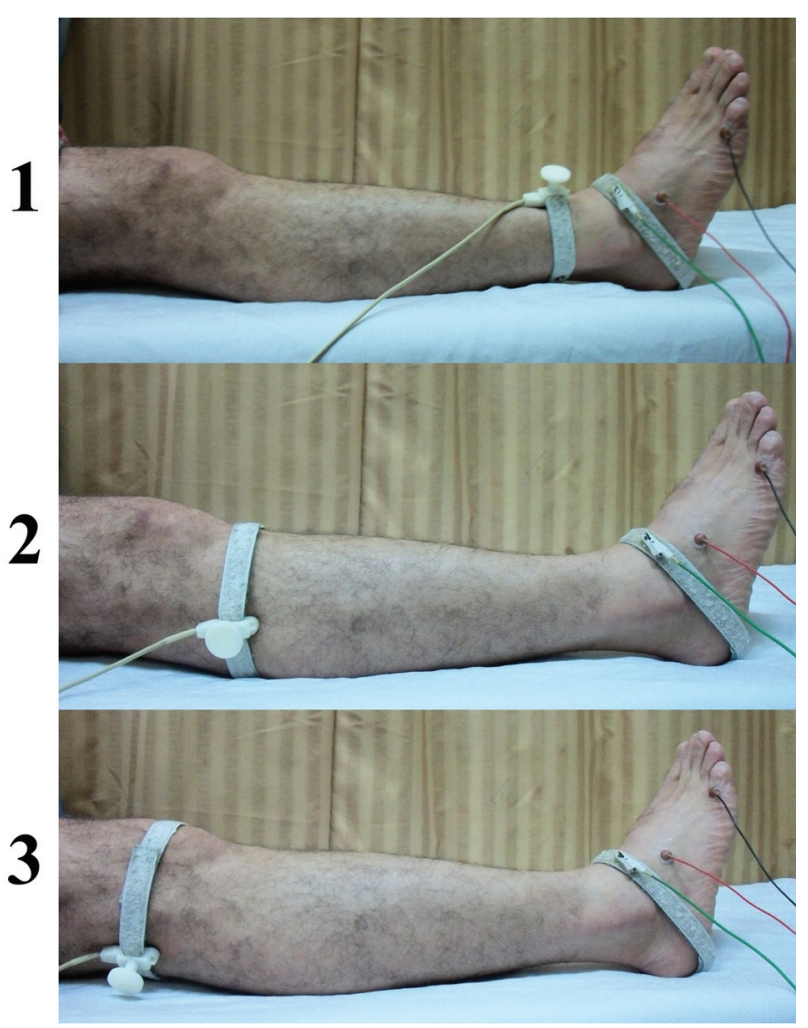

Illustrations of peroneal nerve motor conduction study recording the extensor digitorum brevis muscle: (1) ankle stimulation site, (2) proximal stimulation at the below fibular head site, and (3) proximal stimulation at the lateral popliteal fossa site.

(2) Absent peroneal CMAP while recording from the EDB muscle, stimulating the ankle site, with elicited peroneal CMAP from EDB muscle stimulating proximally.

Electrical stimulation of the ADPN was done behind the lateral malleolus for all the participated subjects (Fig. 3). The sweep speed was $5 \mathrm{~ms} /$ division and the sensitivity was $1-2 \mathrm{mV} /$ division. The CMAP amplitude was taken for the analysis $[1,12,13]$. An elicited response is a confirmation for the presence of ADPN [13].

The ADPN was classified electrophysiologically as the following $[11,13]$ :

(1) ADPN with partial innervation of the EDB muscle: The ADPN supplies the EDB muscle partially. The peroneal CMAP amplitude recorded at the EDB muscle at the ankle stimulation site is lower than the CMAP amplitude obtained at proximal sites of stimulation. This is associated with ADPN CMAP recorded from the EDB muscle.

(2) ADPN with total (exclusive) innervation of the EDB muscle: The ADPN supplies the EDB muscle totally. There is no peroneal CMAP elicited during recording of the EDB muscle at
Figure 3

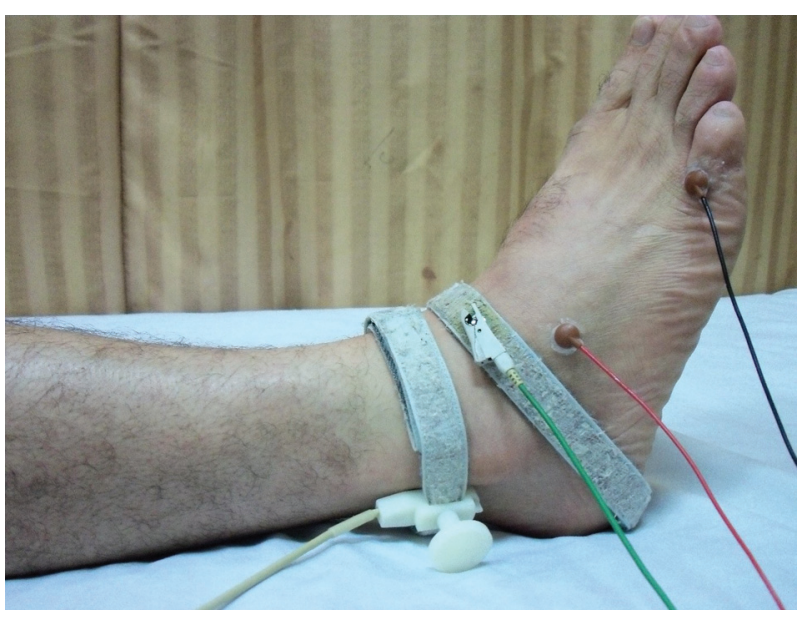

Illustration of accessory deep peroneal nerve motor conduction study. Stimulation is performed behind the lateral malleolus. It is recorded from the extensor digitorum brevis muscle.

the ankle stimulation site, in spite of the presence of CMAP obtained at proximal sites of stimulation. On the other hand, the ADPN CMAP while stimulating behind the lateral malleolus is recorded from the EDB muscle.

Statistical analysis of the results were done by using the statistical package of the social sciences (SPSS version 17) software (University of Cambridge computing service, London, United Kingdom) [14]. Descriptive measures including count, frequency, minimum, maximum, mean, and standard deviation (SD) were used. Analytic measures included Pearson's $\chi^{2}$-test and Fisher's exact when required. Statistical significance was assigned to any $P$ value less than 0.05 .

\section{Results}

The study included 200 lower limbs that were obtained from 100 [56 (56\%) women and 44 (44\%) men] Egyptian apparently healthy volunteers. Their mean age was 39.19 \pm 13.84 years (ranged: $18-75$ years). Electrophysiologically, ADPN was found in $20(10 \%)$ lower limbs of $17(17 \%)$ subjects. Among them, it was present in ten $(58.82 \%)$ women. There was no statistically significant difference between the occurrence of ADPN in women versus men $\left(\chi^{2}=0.066, P=0.797\right)$. ADPN occurred in $11(55 \%)$ right lower limbs and in nine (45\%) left lower limbs. There was no statistically significant difference between the occurrence of ADPN in right lower limbs versus left lower limbs among subjects with $\mathrm{ADPN}\left(\chi^{2}=0.222\right.$, $P=0.637)$.

Among subjects with ADPN, it was present bilaterally in three $(17.65 \%)$ subjects which represent $3 \%$ of the total 
number of subjects included in this study. It was present in two $(66.67 \%)$ women. There was no statistically significant difference between the occurrence of bilateral ADPN in women versus men $\left(\chi^{2}=0.093\right.$, $P=0.761)$.

Among the 14 subjects (82.35\%) with unilateral ADPN, it was present in the right side in eight $(57.14 \%)$ subjects. There was no statistically significant difference between right versus left sides among subjects with unilateral $\operatorname{ADPN}\left(\chi^{2}=0.307, P=0.579\right)$.

All the 17 (100\%) subjects with positive ADPN in our study presented with ADPN with partial innervation of the EDB muscle (Fig. 4). No one showed the ADPN with total innervation of the EDB muscle.

\section{Discussion}

The ADPN arises from the superficial peroneal nerve as a continuation of the muscular branch supplying the

\section{Figure 4}

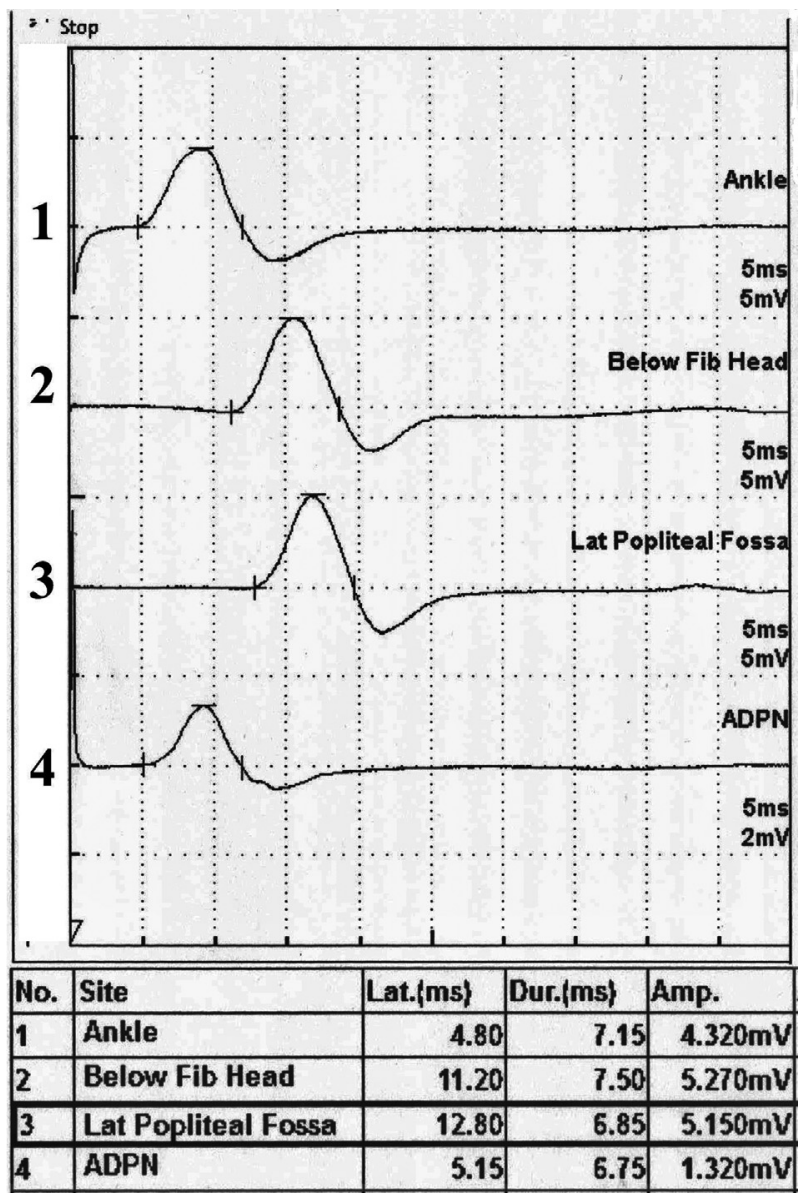

Sample tracings showing the peroneal compound muscle action potentials (CMAP) recording extensor digitorum brevis muscle in a subject with accessory deep peroneal nerve (ADPN). Peroneal CMAP obtained (1) at the ankle, (2) below fibular head, and (3) lateral popliteal fossa stimulation sites; and (4) ADPN CMAP obtained at stimulation behind the lateral malleolus. peroneus longus and peroneus brevis muscles (Fig. 1). It descends in the lateral compartment of the leg along the posterior border of the peroneus brevis muscle. At the ankle region, it passes behind the lateral malleolus in close relation to the sural nerve and deep into the peroneus brevis tendon to reach the dorsum of the foot $[10,15]$. In this region, it gives deep sensory supply to the ankle joint and the surrounding ligaments and tendons. Also, it gives motor branch to supply the lateral aspect of EDB muscle [15]. However, total innervation of the EDB muscle by the ADPN could occur [11,15-17].

Peripheral nerves anomalous innervations are important aspects in routine neurophysiological assessment of any patient. Unrecognition of these anomalous innervations can be mistaken for technical pitfalls or for actual pathology $[1,18]$. It is important to take into consideration the anatomic variations in the innervations of muscles during electrophysiological assessment of nerves $[4,19]$.

The prevalence of ADPN in a sample of Egyptian subjects was $17 \%$ electrophysiologically (10\% of the examined lower limbs). The prevalence of ADPN in this study was within the range of prevalence of ADPN present in other studies which varies from 12 to $35 \%$ among the studied subjects $(8.2-27 \%$ of the studied lower limbs) [10]. It was found that there is a wide variation of prevalence of ADPN among different studies [2,12,13,20,21]. A meta-analysis study assessed the prevalence of ADPN and found the following: (i) the overall pooled prevalence was $18.8 \%$ of lower limbs; (ii) the electrophysiological pooled prevalence was $13.6 \%$; and (iii) the anatomical pooled prevalence was $39.3 \%$ [2]. This could be explained by the differences between studies regarding the studied population and the techniques used in the assessment of ADPN, whether anatomical or electrophysiological studies [2]. The anatomical studies showed a higher prevalence of ADPN than that obtained by electrophysiological studies. It was found that when the ADPN supplies the EDB muscle by a motor branch is the only situation that can be detected electrophysiologically. However, if the ADPN has no motor supply to the EDB muscle, it could not be detected except anatomically $[2,10]$. It was reported that the pooled prevalence of ADPN with motor supply to the EDB muscle was $16.3 \%$ anatomically, which is approximately equal to the electrophysiological pooled prevalence of ADPN [2].

In this research, there was no statistically significant difference between women and men as regards the 
frequency of ADPN. This was in agreement with other studies $[12,13]$. This could be due to the autosomal dominant inheritance of ADPN. It was reported to be about three times more common in the family members of persons who had ADPN $[8,20]$.

In this study, there was no statistically significant difference between the occurrence of ADPN in right lower limbs versus left lower limbs among subjects with ADPN. This was in accordance with other studies $[2,10,12,13,20]$. In this study, unilateral ADPN was present in $82.35 \%$ of the subjects with ADPN. This was in agreement with previous studies which reported that unilateral ADPN was more common than bilateral ADPN $[2,12,13]$.

Regarding the electrophysiological forms of ADPN, ADPN with partial innervation of the EDB muscle was the only form to be present. It is the form in which the ADPN supplies the lateral part of the EDB muscle, while its medial part is supplied by the deep peroneal nerve. All our 17 subjects with recorded ADPN had ADPN with partial innervation of the EDB muscle. This result was similar to previous studies which reported that ADPN with total innervation of the EDB muscle is a rare condition with the presence of few case reports describing it $[11,16,17]$. It was postulated that the calculated incidence of total innervation of the $\mathrm{EDB}$ muscle by the $\mathrm{ADPN}$ is about $0.49 \%$ [11].

The ADPN has more than one clinical importance $[2,10]$. Studying the ADPN can complicate the clinical picture and disturb the interpretation of the electrophysiological studies conducted during the assessment of common peroneal, deep peroneal, and superficial peroneal nerves lesions and injuries, as well as, ADPN neuropathy $[1,2,10,13,22-24]$.

The presence of ADPN with deep peroneal nerve lesion could give the picture of an incomplete deep peroneal nerve lesion instead of a complete one. In this case, there is partial preservation of the function of the EDB muscle in spite of complete loss of function of the tibialis anterior muscle. In this situation, the peroneal CMAP at the ankle stimulation site is unelicited while it is elicited proximally. Needle electromyography (EMG) of the $\mathrm{EDB}$ muscle shows evidence of partial nerve lesion $[2,3,10,13]$. In case of superficial peroneal nerve lesion, the presence of ADPN could give the picture of common peroneal nerve lesion instead of superficial peroneal nerve lesion $[2,3,10,13]$. In this case, there is weakness of the EDB muscle with weakness of the peroneus longus and brevis muscles in spite of complete preservation of the tibialis anterior muscle. Electrophysiologically, the peroneal CMAP elicited by distal and proximal stimulation sites could be less than normal. Needle EMG of the peroneus longus, peroneus brevis, as well as EDB muscles show an abnormal EMG pattern, while the tibialis anterior muscle shows normal EMG pattern $[2,3,10,13]$. It is difficult to diagnose cases with partial lesion of the common peroneal nerve with focal conduction block at the transfibular segment having ADPN. The small CMAP obtained at the lateral popliteal fossa stimulation site could be similar to that obtained at the ankle stimulation site. In this situation, the CMAP obtained at below the fibular head site of stimulation could be larger than that obtained in the other two sites of stimulation [19].

Entrapment neuropathy of ADPN could be a cause of chronic ankle pain. The entrapment could be due to compression of the ADPN between peroneus accessories (peroneus quartus) muscle (a common variant muscle of the ankle) and peroneus brevis tendon in the lateral ankle region behind the lateral malleolus $[2,15,24,25]$.

Iatrogenic injury to the ADPN during surgery could be occurred if the surgeon did not put ADPN presence into his consideration $[10,15]$. This could occur during orthopedic surgery in the ankle region which is usually done through a lateral approach in which the incision is behind the lateral malleolus where the ADPN could be located. Also, this could occur during sural nerve biopsy due to the close relationship between the ADPN and the sural nerve $[10,15]$. The surgeon should be aware of the presence of ADPN which could be injured during these procedures if it is present $[2,10]$. ADPN injury could lead to pain in the posterolateral aspect of the ankle region [24]. So, it is recommended to assess the presence of ADPN before doing any surgery in the ankle region and sural nerve biopsy $[2,10]$.

This study had a sampling limitation as it was conducted only in one medical center in one Egyptian governorate. It was difficult to generalize the results of this study to all Egyptian population. Further studies are recommended on a larger scale of Egyptian subjects from different governorates for proper and wider calculation of the prevalence of ADPN among Egyptian population.

\section{Conclusion}

This study demonstrated that ADPN prevalence in the referred Egyptian sample through electrodiagnostic 
studies of lower limbs was $17 \%$, with no sex nor side difference. Recognition of ADPN is essential for proper interpretation of lower limb electrophysiological data, which avoids the error in the diagnosis of common peroneal, deep peroneal, and superficial peroneal nerve lesions.

\section{Acknowledgements}

The author acknowledges Maria K. Saba for her drawings and technical assistance in the preparation of figures especially Figure 1.

\section{Financial support and sponsorship}

Nil.

\section{Conflicts of interest}

There are no conflicts of interest.

\section{References}

1 Preston D, Shapiro B. Electromyography and neuromuscular disorders: clinical-electrophysiologic correlations. 3rd ed. Pennsylvania, PA: Elsevier Saunders; 2013.

2 Tomaszewski KA, Roy J, Vikse J, Pekala PA, Kopacz P, Henry BM. Prevalence of the accessory deep peroneal nerve: a cadaveric study and meta-analysis. Clin Neurol Neurosurg 2016; 144:105-111.

3 Dessi F, Durand G, Hoffmann JJ. The accessory deep peroneal nerve: a pitfall for the electromyographer. J Neurol Neurosurg Psychiatry 1992; 55:214-215.

4 Kuruvilla A. Accessory deep peroneal nerve. Neurol India 2004; 52:135.

5 Neundörfer B, Seiberth R. The accessory deep peroneal nerve. J Neurol 1975; 209:125-129.

6 Masakado Y, Kawakami M, Suzuki K, Abe L, Ota T, Kimura A. Clinical neurophysiology in the diagnosis of peroneal nerve palsy. Keio J Med 2008; 57:84-89.

7 Baima J, Krivickas L. Evaluation and treatment of peroneal neuropathy. Curr Rev Musculoskelet Med 2008; 1:147-153.
8 Owsiak S, Kostera-Pruszczyk A, Rowińska-Marcinska K. Accessory deep peroneal nerve - a clinically significant anomaly? Neurol Neurochir Pol 2008; 42:112-115.

9 Infante E, Kennedy WR. Anomalous branch of the peroneal nerve detected by electromyography. Arch Neurol 1970; 22:162-165.

10 Tzika M, Paraskevas GK, Kitsoulis P. The accessory deep peroneal nerve: a review of the literature. Foot (Edinb) 2012; 22:232-234.

11 Murad H, Neal P, Katirji B. Total innervation of the extensor digitorum brevis by the accessory deep peroneal nerve. Eur J Neurol 1999; 6:371-373.

12 Mathis S, Ciron J, du Boisguéheneuc F, Godenèche G, Hobeika L, Larrieu $D$, et al. Study of accessory deep peroneal nerve motor conduction in a population of healthy subjects. Neurophysiol Clin 2011; 41:29-33.

13 Rayegani SM, Daneshtalab E, Bahrami MH, Eliaspour D, Raeissadat SA, Rezaei $\mathrm{S}$, et al. Prevalence of accessory deep peroneal nerve in referred patients to an electrodiagnostic medicine clinic. J Brachial Plex Peripher Nerve Inj 2011; 6:3.

14 Statistical Package of Social Science, version 17 London: University of Cambridge computing service. Documentation: 2007

15 Kudoh H, Sakai T, Horiguchi M. The consistent presence of the human accessory deep peroneal nerve. J Anat 1999; 194:101-108.

16 Kayal R, Katirji B. Atypical deep peroneal neuropathy in the setting of an accessory deep peroneal nerve. Muscle Nerve 2009; 40:313-315.

17 Ubogu EE. Complete innervation of extensor digitorum brevis by accessory peroneal nerve. Neuromuscul Disord 2005; 15:562-564.

18 Saba EK. Electrophysiological study of Martin-Gruber anastomosis in a sample of Egyptians. Egypt Rheumatol Rehabil 2017; 44:153-158.

19 Gutmann L. AAEM mini-monograph \#2: important anomalous innervations of the extremities. Muscle Nerve 1993; 16:339-347.

20 Crutchfield CA, Gutmann L. Hereditary aspects of accessory deep peroneal nerve. J Neurol Neurosurg Psychiatry 1973; 36:989-990.

21 Budak F, Gönenc Z. Innervation anomalies in upper and lower extremities (an electrophysiological study). Electromyogr Clin Neurophysiol 1999; 39:231-234

22 Sinanović O, Zukić S, Sakić A, Muftić M. The accessory deep peroneal nerve and anterior tarsal tunnel syndrome: case report. Acta Myol 2013; 32:110-112.

23 Koo YS, Cho CC, Kim BJ. Pitfalls in using electrophysiological studies to diagnose neuromuscular disorders. J Clin Neurol 2012; 8:1-14.

24 Sander HW, Quinto C, Chokroverty S. Accessory deep peroneal neuropathy: collision technique diagnosis. Muscle Nerve 1998; 21:121-123

25 Clarkson MJ, Fox JN, Atsas S, Daney BT, Dodson SC, Lambert HW. Clinical implications of novel variants of the fibularis (peroneus) quartus muscle inserting onto the cuboid bone: peroneocuboideus and peroneocalcaneocuboideus. J Foot Ankle Surg 2013; 52:118-121. 